\title{
Relación entre la adherencia al tratamiento antirretroviral en pacientes VIH+ y el consumo de alcohol, asociado o no al uso de otras sustancias
}

\author{
Relationship between alcohol consumption, whether \\ linked to other substance use or not, and antiretroviral \\ treatment adherence in HIV+ patients
}

\author{
Sara González-Álvarez*; Agustín Madoz-Gúrpide**; Carloos Parro-Torres***; Daniel \\ Hernández-Huerta*; Enriqueta Ochoa Mangado****. \\ * Servicio de Psiquiatría, Hospital Universitario Ramón y Cajal. Madrid, Spain; ** Centro de Salud Mental San Blas. Servicio de \\ Psiquiatría, Hospital Universitario Ramón y Cajal (IRYCIS). Universidad de Alcalá. Madrid, Spain; *** Servicio de Psiquiatría, \\ Hospital General Universitario Gregorio Marañón. Madrid, Spain; **** Servicio de Psiquiatría, Hospital Universitario Ramón y \\ Cajal (IRYCIS). Universidad de Alcalá. Madrid, Spain.
}

\section{Resumen}

El consumo perjudicial de alcohol es un diagnóstico de elevada prevalencia en pacientes VIH+. Distintos estudios han destacado la influencia negativa del mismo sobre la adherencia al tratamiento antirretroviral, aunque pocos de ellos valoran además el consumo de otras sustancias. En España, el consumo de alcohol se presenta frecuentemente en situación de policonsumo, fundamentalmente de cannabis y cocaína. El objetivo es comprobar cómo influye el consumo de alcohol, asociado o no al uso de otras sustancias (cocaína, heroína, metadona y/o cannabis), en la adherencia al tratamiento antirretroviral en nuestro entorno. Se ha realizado un estudio observacional tipo casos y controles sobre una muestra de 119 individuos VIH+. Conforman los casos $(n=40)$ sujetos no adherentes al tratamiento farmacológico según reporte de Farmacia Hospitalaria, corroborado por el Simplified Medication Adherence Questionnaire (SMAQ) y la opinión del profesional de referencia. Se consideran controles $(n=79)$ una muestra de pacientes de características similares con buena adherencia terapéutica según los mismos métodos de valoración. La recogida de datos se hizo entre mayo 2013 y septiembre 2015. El análisis estadístico se realizó mediante regresión logística binaria. Los resultados muestran que el consumo de alcohol empeora la adherencia al tratamiento antirretroviral. El uso de metadona supone un incremento estadísticamente significativo del riesgo de no adherencia. No se han encontrado diferencias significativas entre los grupos del estudio respecto a los consumos de cocaína, heroína o cannabis. Por tanto, la detección del consumo de sustancias, especialmente de alcohol, y su abordaje en pacientes $\mathrm{VIH}+$ puede repercutir positivamente en el cumplimiento terapéutico, en beneficio de una mayor efectividad de la terapia antirretroviral. Palabras clave: VIH; Terapia antirretroviral (TARGA); Adherencia terapéutica; Trastorno por uso de alcohol; Policonsumo.

\begin{abstract}
Hazardous alcohol consumption is a common diagnosis among people living with HIV infection. The relationship between alcohol consumption and poor adherence to antiretroviral therapy has been highlighted in different studies, yet few of them performed a parallel analysis of other substance use. In Spain, alcohol consumption is frequently associated with other substance use, mainly cannabis and cocaine. The aim of this study is to assess the influence of hazardous alcohol consumption both combined with other substances (cocaine, heroin, methadone and/or cannabis) or alone on antiretroviral therapy adherence in our social environment. We performed an observational case-control study including 119 HIV+ individuals. We recruited 40 non-adherent patients, defined by less than $90 \%$ compliance according to hospital pharmacy refill data, and corroborated by the Simplified Medication Adherence Questionnaire (SMAQ) and referring professional's opinion. Control cases $(n=79)$ were defined as those patients with similar characteristics but considered adherent according to the same parameters. Data collection took place between May 2013 and September 2015. Statistical analysis was performed using a binary logistic regression model. Our results indicate that alcohol consumption decreases adherence to antiretroviral therapy. The use of methadone represents a statistically significant increased risk of poor adherence. No significant differences were found between adherent and non-adherent groups regarding cocaine, heroin or cannabis use in this study. In summary, the detection of substance use and especially alcohol consumption in HIV+ patients can improve the effectiveness of antiretroviral therapy by identifying and treating at-risk individuals for a poor therapeutic adherence.
\end{abstract}

Keywords: HIV; Highly Active Antiretroviral Therapy (HAART); Medication adherence; Alcohol use; Substance use. 
$\mathrm{E}$ 1 consumo perjudicial de alcohol y otras sustancias es un diagnóstico frecuente en pacientes con infección por VIH. Diversos autores han señalado su elevada prevalencia (Parsons, Starks, Millar, Boonrai y Marcotte, 2014; Skalski, Sikkema, Heckman y Meade, 2013). Aunque las estimaciones varían entre el $8 \%$ y el $42 \%$ (Williams et al., 2016), se ha señalado que la prevalencia de consumo intensivo de alcohol (heavy drinking) (Cortés Tomás y Motos Sellés, 2016) en estos pacientes casi alcanza a duplicar la prevalencia en la población general (Galvan et al., 2002).

Entre las consecuencias del consumo de alcohol en pacientes $\mathrm{VIH}+$ se han señalado, además del perjuicio directo sobre la propia salud individual, otros factores como el aumento potencial del riesgo de transmisión del virus en situaciones de intoxicación etílica (Scott-Sheldon et al., 2013), el retraso en el diagnóstico de la enfermedad (Zarkin, Bray, Babor y Higgins-Biddle, 2004), un incremento en el riesgo de deterioro cognitivo temprano (Anand, Springer, Copenhaver y Altice, 2010) o de contagio por virus de la hepatitis C (Taylor, Denniston, Klevens, McKnight-Eily y Jiles, 2016) y, en general, un aumento de la morbimortalidad (Azar, Springer, Meyer y Altice, 2010). Además, el consumo perjudicial de alcohol se ha relacionado con un empeoramiento del estado inmunológico de forma independiente al tratamiento (Baum et al., 2010).

En España, las tasas de nuevos diagnósticos de VIH continúan siendo superiores a la media de los países de la Unión Europea y de Europa Occidental. Durante 2015, se estimaron alrededor de 9,44 nuevos casos por cada 100.000 habitantes (Instituto de Salud Carlos III, 2016). Desde la instauración del tratamiento antirretroviral de gran actividad (TARGA) como terapia de elección en la infección por VIH, se ha producido una mejora significativa en la calidad y esperanza de vida de los individuos afectados (Tancredi y Waldman, 2014; Poorolajal, Hooshmand, Mahjub, Esmailnasab y Jenabi, 2016). Los beneficios de dicha pauta, no obstante, están íntimamente ligados al estricto cumplimiento terapéutico, habiéndose postulado la falta de adherencia al mismo como la primera causa de fracaso del tratamiento a corto plazo (Braithwaite y Bryant, 2010), además de favorecer el desarrollo de cepas víricas resistentes (Cohen, 2006).

Existen numerosas líneas de investigación en relación a los factores que pueden incidir de forma negativa en la adherencia al tratamiento antirretroviral. Entre ellos, se han resaltado la presencia de efectos no deseados del tratamiento, el estrés emocional secundario a problemática vital, la ausencia de soporte sociofamiliar, la complejidad de la pauta farmacológica o la falta de eficacia percibida por parte de los pacientes (Ammassari et al., 2002; Hudelson y Cluver, 2015). Así mismo, se han asociado con un peor cumplimiento terapéutico la edad joven o el deterioro cognitivo (Hinkin et al., 2004), la presencia de psicopatología, fundamentalmente depresiva (Palepu, Horton, Tibbetts, Meli y Samet, 2004) así como determinados rasgos de personalidad (Hutton y Treisman, 2008), un nivel socioeconómico bajo (Bermudez et al., 2016; Peltzer y Pengpid, 2013) y el consumo de sustancias, en particular de alcohol (Azar et al., 2010; Palepu et al., 2004).

Se ha postulado que la adherencia al tratamiento antirretroviral debe entenderse como un proceso dinámico en el que los factores de riesgo modificables se asociarían con la mejora o el empeoramiento de la misma (Lazo et al., 2013). En los últimos años, numerosos autores han prestado especial atención a las repercusiones del consumo de alcohol sobre el cumplimiento terapéutico (Vagenas et al., 2015). Diferentes revisiones sistemáticas sustentan de manera consistente la relación entre ambos factores (Azar et al., 2010; Vagenas et al., 2015; Hendershot, Stoner, Pantalone y Simoni, 2009). Además, el consumo de alcohol u otras sustancias puede influir también en la adherencia a otros tratamientos médicos relacionados con enfermedades o infecciones comunes en pacientes VIH+ (Gonzalez, Barinas y O'Cleirigh, 2011). Consecuentemente, se han propuesto programas de intervención terapéutica para disminuir la ingesta de alcohol y mejorar el nivel de cumplimiento farmacológico, lo que se traduciría en una mejora global en el curso de la enfermedad por VIH (Parry et al., 2014). En esta línea, se ha señalado que el paso de la situación de consumo activo de sustancias a la abstinencia estaría consistentemente asociado con el fortalecimiento de la adherencia terapéutica (Lucas, Gebo, Chaisson y Moore, 2002).

En España, el consumo de alcohol se asocia frecuentemente al de otras sustancias. Según la Encuesta Domiciliaria sobre Alcohol y Drogas en España, publicada por el Ministerio de Sanidad en 2015 (OEDT, 2015), el alcohol está presente en la práctica totalidad de las situaciones de policonsumo: el 94,3\% de los consumidores de cannabis y el $96,8 \%$ de los consumidores de cocaína presentan consumo de alcohol concomitante. Además, entre los bebedores de alcohol como sustancia principal, el 11,1\% presenta también consumo de cannabis, y el 2,7\% de cocaína.

Existe evidencia de que la influencia sobre la adherencia terapéutica puede variar en función de la sustancia consumida y del patrón de consumo (Azar et al., 2015; Gonzalez et al., 2011), aunque los tipos de sustancia y los mecanismos que relacionan su uso con el empeoramiento de la adherencia no han sido estudiados en profundidad (Gonzalez, Mimiaga, Israel, Andres Bedoya y Safren, 2013). En un estudio llevado a cabo por Parsons y colaboradores (2014), sobre una muestra de 557 individuos VIH+ mayores de 50 años, el uso combinado de alcohol y cannabis o cocaína se asoció de manera estadísticamente significativa con un aumento de tomas olvidadas respecto al consumo aislado de alcohol.

El cumplimiento terapéutico requiere una atención meticulosa a los tiempos de las dosis y, en muchos casos, la coordinación de múltiples medicaciones, implicando me- 
canismos relacionados con la cognición, la planificación y la toma de decisiones. Elementos como la inestabilidad en el estilo de vida (Tucker et al., 2004), la vulnerabilidad física y psíquica, o las dificultades en el autocontrol mediadas por el consumo de sustancias podrían interactuar en detrimento de la adherencia. Además, algunos pacientes pueden interrumpir el tratamiento durante episodios de consumo al percibirlo como incompatible con el uso de sustancias (Kalichman et al., 2015).

A pesar de lo expuesto, basado en datos internacionales, las referencias en la literatura sobre la repercusión del consumo alcohol en la adherencia al tratamiento antirretroviral en pacientes VIH+ en España son limitadas (Ortego, Huedo-Medina, Vejo y Llorca, 2011; Pérez-Valero et al., 2016a; Pérez-Valero et al., 2016b). El objetivo del presente estudio es valorar si el consumo perjudicial de alcohol se relaciona con un peor cumplimiento de la terapia antirretroviral en pacientes VIH+ una vez ajustado por el consumo de otras sustancias (cocaína, heroína, metadona y/o cannabis), y conocer el papel que éstas juegan en la adherencia en un entorno asistencial concreto y en el contexto cultural de la población española.

\section{Metodología}

\section{Sujetos}

Conforman la población de estudio pacientes adultos en seguimiento regular de forma ambulatoria en consultas del servicio de Enfermedades Infecciosas de un hospital de tercer nivel (Hospital Universitario Ramón y Cajal, Madrid), a los que les estaba indicado durante al menos los doce meses anteriores TARGA, dispensado en todos los casos a través de Farmacia Hospitalaria. Todos ellos firmaron un consentimiento informado antes de participar en el estudio. Se consideró seguimiento regular el haber acudido al menos a dos citas concertadas en el último año (Tripathi, Youmans, Gibson y Duffus, 2011). Se consideraron criterios de exclusión la presencia de procesos infecciosos agudos u oncológicos activos, además del rechazo a participar o la retirada voluntaria del estudio en cumplimiento del consentimiento informado. Además, se excluyeron del estudio aquellos casos en los que existiese incongruencia entre los resultados del cuestionario SMAQ y/o la opinión del profesional de referencia respecto a la adherencia según Farmacia Hospitalaria. El estudio fue aprobado por el Comité Ético de Investigación Clínica del Hospital antes de llevarse a cabo.

\section{Procedimiento}

Se trata de un estudio observacional tipo casos y controles. Conforman los casos pacientes no adherentes a TARGA; y los controles, una muestra de pacientes de características similares con buena adherencia terapéutica. La selección de los casos se realizó a través de un muestreo consecutivo de un listado de pacientes no adherentes ge- nerado de forma ordinaria y con frecuencia mensual por Farmacia Hospitalaria. Los controles fueron seleccionados por muestreo consecutivo del listado de pacientes con cita en Consultas Externas. Se consideraron pacientes no adherentes aquellos que, según reporte de Farmacia Hospitalaria, hubiesen retirado menos del $90 \%$ de las tomas del tratamiento antirretroviral pautado durante el último año. Se consideraron pacientes adherentes aquellos que presentasen un $95 \%$ o más en la retirada de las tomas durante el último año según el Servicio de Farmacia Hospitalaria (Paterson et al., 2000). Se excluyeron del estudio aquellos pacientes cuya adherencia fuera igual o mayor que $90 \%$ pero menor de $95 \%$, dado que no existe unanimidad en la literatura sobre los límites de esta diferenciación (Viswanathan et al., 2015). A todos los individuos participantes en el estudio se les aplicó una entrevista clínica semi-estructurada de evaluación elaborada ad hoc para el mismo. En ella se incluyó el cuestionario SMAQ -instrumento breve basado en seis preguntas al propio paciente en relación a su cumplimiento terapéutico, validado para su uso en pacientes con infección por VIH (Knobel et al., 2002)- para corroborar la valoración de adherencia terapéutica según Farmacia Hospitalaria. Todo el proceso de evaluación fue llevado a cabo por los investigadores. La recogida de datos se hizo entre mayo 2013 y septiembre 2015.

\section{Variables del estudio}

Se incluyeron variables sociodemográficas como edad, sexo, raza, estado civil, nivel socioeconómico, educativo y laboral, entre otras. Además, se realizó un registro de variables del estado de la infección por VIH y su tratamiento tales como tiempo de evolución desde el inicio del seguimiento y del tratamiento, número de pastillas y de tomas de tratamiento antirretroviral al día, así como la presencia o no de una figura de supervisión de la toma del mismo. En cuanto al uso de sustancias, se recogió historia de consumo de alcohol, cannabis, cocaína, heroína y metadona (uso terapéutico) en los 12 meses previos a la valoración. De todas ellas se tuvieron en cuenta la frecuencia de consumo y la cantidad diaria consumida.

\section{Análisis estadístico}

En el análisis estadístico se obtuvieron los valores descriptivos y las frecuencias de las variables incluidas en el estudio. Algunas de las variables fueron recodificadas para no perder potencia en el análisis, sin que ello supusiera pérdida sustancial de información y preservando un sentido clínico. Fueron categorizadas como nivel socioeconómico problemático o de riesgo situaciones de dificultad económica con o sin ayuda externa, imposibilidad para amortizar pagos o deudas o riesgo de desahucio. El consumo de alcohol fue categorizado de forma dicotómica en consumo no problemático y consumo perjudicial, según los criterios establecidos por la Organización Mundial de la Sa- 
lud (O.M.S., 1992). Para el resto de sustancias, se incluyó como positivo el uso continuado de la sustancia a estudio, cualquiera que fuera su patrón de consumo (todos los individuos presentaban frecuencia al menos semanal). En la descripción de variables cuantitativas, que no cumplían criterios de normalidad de la distribución, se eligió la mediana y el rango intercuartil (IQ25-75). Para el análisis comparativo de datos brutos, en ese caso se empleó la prueba de U de Mann-Whitney. En el caso de variables cualitativas se recurrió al estadístico chi-cuadrado de Pearson. En ambos casos se seleccionó un nivel de significación p=0,05.

Por último, se llevó a cabo el análisis multivariante mediante regresión logística binaria. Se empleó el método «Introducir» (Enter) en la inclusión de variables predictoras en la ecuación. Se incluyeron en el modelo las variables que habían demostrado en el análisis bruto relación con la adherencia al tratamiento, y que centran el objetivo de nuestro estudio en relación al consumo de sustancias, junto con aquellas otras variables recomendadas por la literatura especializada. Se incluyen en dicho análisis: edad, género, nivel socioeconómico, tiempo de seguimiento, número de pastillas prescritas al día, y consumo de alcohol, cannabis, cocaína, heroína y metadona.

\section{Resultados}

\section{Resultados descriptivos y análisis bruto}

Características sociodemográficas y clínicas de la muestra.

Se analizó una muestra total de 119 sujetos, siendo 40 de ellos casos y 79 controles. La mediana de edad del conjunto de la población fue de 48,5 (IQ: 44,3-52,8). El tiempo en seguimiento y el tiempo en tratamiento, ambos en años,
Tabla 1. Variables sociodemográficas del conjunto de la muestra.

\begin{tabular}{llcc}
\hline \multirow{2}{*}{ Género } & & $\mathbf{n}$ & \multicolumn{1}{c}{$\%$} \\
\hline \multirow{2}{*}{ Raza } & Varón & 79 & $66,4 \%$ \\
\cline { 2 - 4 } & Mujer & 40 & $33,6 \%$ \\
\hline \multirow{2}{*}{ Estado civil } & Caucásica & 99 & $83,2 \%$ \\
\cline { 2 - 4 } & Otras & 20 & $16,8 \%$ \\
\hline \multirow{2}{*}{ Trabajo } & En pareja & 46 & $38,7 \%$ \\
\cline { 2 - 4 } & Otros & 73 & $61,3 \%$ \\
\hline \multirow{2}{*}{ Profesión } & Sí trabaja & 53 & $44,5 \%$ \\
\cline { 2 - 4 } & No trabaja & 66 & $55,5 \%$ \\
\hline \multirow{2}{*}{ Nivel socioeconómico } & No cualificada & 54 & $46,2 \%$ \\
\cline { 2 - 4 } & Cualificada & 63 & $53,8 \%$ \\
\hline \multirow{2}{*}{ Supervisión tratamiento } & Estable & 90 & $75,6 \%$ \\
\cline { 2 - 4 } & Riesgo & 29 & $24,4 \%$ \\
\cline { 2 - 4 } & Nunca & 98 & $82,4 \%$ \\
\hline
\end{tabular}

Tabla 2. Variables de consumo del conjunto de la muestra.

\begin{tabular}{lcc}
\hline & Sí & No \\
\cline { 2 - 3 } & $\mathrm{n}(\%)$ & $\mathrm{n}(\%)$ \\
\hline Consumo Alcohol & $27(23,3)$ & $89(76,7)$ \\
\hline Consumo Cannabis & $39(32,8)$ & $80(67,2)$ \\
\hline Consumo Cocaína & $29(24,4)$ & $90(75,6)$ \\
\hline Consumo Heroína & $8(6,7)$ & $111(93,3)$ \\
\hline Consumo Metadona & $17(14,3)$ & $102(85,7)$ \\
\hline Policonsumo & $36(30,3)$ & $83(69,7)$ \\
\hline
\end{tabular}

Tabla 3. Variables sociodemográficas y significación estadística de la comparación. Casos y controles.

\begin{tabular}{|c|c|c|c|c|c|}
\hline & & \multirow{2}{*}{$\begin{array}{c}\text { Controles } \\
\mathrm{n}(\%)\end{array}$} & \multirow{2}{*}{$\begin{array}{l}\text { Casos } \\
\mathrm{n}(\%)\end{array}$} & \multicolumn{2}{|c|}{ Prueba de Pearson } \\
\hline & & & & $\chi^{2}$ & Sig. \\
\hline \multirow{2}{*}{ Género } & Varón & $52(65,8)$ & $27(67,5)$ & \multirow{2}{*}{0,033} & \multirow{2}{*}{0,855} \\
\hline & Mujer & $27(34,2)$ & $13(32,5)$ & & \\
\hline Raza & Caucásica & $70(88,6)$ & $29(72,5)$ & 4,928 & 0,026 \\
\hline \multirow{2}{*}{ Estado civil } & En pareja & $28(35,4)$ & $18(45,0)$ & \multirow{2}{*}{1,023} & \multirow{2}{*}{0,312} \\
\hline & Otros & $51(64,6)$ & $22(55,0)$ & & \\
\hline Situación laboral & No trabaja & $41(51,9)$ & $25(62,5)$ & 1,208 & 0,272 \\
\hline \multirow[b]{2}{*}{ Profesión } & No cualificada & $31(40,3)$ & $23(57,5)$ & \multirow{2}{*}{3,148} & \multirow{2}{*}{0,312} \\
\hline & Cualificada & $46(59,7)$ & $17(42,5)$ & & \\
\hline \multirow{2}{*}{ Nivel socioeconómico } & Estable & $69(87,3)$ & $21(52,5)$ & \multirow{2}{*}{17,490} & \multirow{2}{*}{0,000} \\
\hline & Riesgo & $10(12,7)$ & $19(47,5)$ & & \\
\hline \multirow{2}{*}{ Supervisión tratamiento } & Nunca & $71(89,9)$ & $27(67,5)$ & \multirow{2}{*}{9,146} & \multirow{2}{*}{0,002} \\
\hline & A veces/siempre & $8(10,1)$ & $13(32,5)$ & & \\
\hline
\end{tabular}


presentaban una mediana (IQ) respectiva de 16,5 (9-21) y 15 (7-19). El conjunto de sujetos tomaba como mediana 3 pastillas/d (IQ: 1-3). En la Tabla 1 y 2 se presentan otros datos cualitativos que caracterizan la muestra global.

Son varones 52 individuos de los controles $(65,8 \%)$ y 27 de los casos $(67,5 \%)$ de la muestra (Tabla 3$)$. La mediana de edad de la muestra es de 47,6 (42,5-50,6) en el grupo de casos y de 49,1 (45,3-53,5) en el grupo control, con una mediana de seguimiento en años en el grupo de casos de 17,5 $(9,8-22,0)$ y de $15,0(8,0-21,0)$ en los controles (Tabla 4$)$.

En el análisis comparativo, se evidencia de manera estadísticamente significativa el mayor estatus socioeconómico del grupo control. Asimismo, los casos presentan mayor grado de supervisión del tratamiento por parte de terceros (Tabla 3). Respecto a las variables cuantitativas, entre casos y controles solo se encontraron diferencias estadísticamente significativas en el número de pastillas al día (Tabla 4).

\section{Variables relacionadas con el uso de sustancias.}

Un total de 15 sujetos $(37,5 \%)$ presentaban consumo perjudicial de alcohol en la muestra constituida por los casos, frente a 12 sujetos $(15,8 \%)$ en el grupo control $(\mathrm{p}=0,009)$.

Como muestra el análisis de variables cualitativas, los casos presentan un perfil caracterizado por mayores con- sumos de cada sustancia valorada salvo de cannabis, donde no hay diferencias respecto a los controles. Se objetivó uso terapéutico de metadona en 12 individuos entre los casos $(30,0 \%)$, en contraste con 5 sujetos del grupo control $(6,3 \%)(p=0,000)$. La diferencia para consumo de cocaína resultó también estadísticamente significativa $(\mathrm{p}=0,005)$. En el caso de la heroína, existirían diferencias entre ambos grupos con una $\mathrm{p}=0,073$ (Tabla 5).

\section{Regresión logística}

En la Tabla 6 se muestran los resultados del análisis de regresión logística, donde se resaltan las variables que muestran influencia sobre la adherencia terapéutica de manera estadísticamente significativa. El modelo aplicado clasifica correctamente a cerca del $80 \%$ de los sujetos, con mayor especificidad que sensibilidad (prueba de Hosmer y Lemeshow: $\operatorname{casos}=55,7 \%$, controles $=90,0 \%$, global $=78,3 \%$ ) y explica el 44,2\% de variabilidad de la adherencia.

\section{Consumo de sustancias.}

De los datos expuestos se deduce que el consumo perjudicial de alcohol supone un incremento medio de 4,330 (IC95\%: 1,157-16,206) del riesgo de mala adherencia al tratamiento antirretroviral de manera estadísticamente significativa $(p=0,030)$ frente a la situación de abstinencia

Tabla 4. Variables clínicas y significación estadística de la comparación. Casos y controles.

\begin{tabular}{|c|c|c|c|c|c|c|}
\hline & & \multirow{2}{*}{ n } & \multirow{2}{*}{ Mediana } & \multicolumn{2}{|c|}{ Rango intercuartílico } & \multirow{2}{*}{$\begin{array}{c}\begin{array}{c}\text { U de Mann } \\
\text { Whitney }\end{array} \\
\text { Sig. }\end{array}$} \\
\hline & & & & Q25 & Q75 & \\
\hline \multirow{2}{*}{ Edad } & Casos & 40 & 47,66 & 42,50 & 50,61 & \multirow{2}{*}{0,086} \\
\hline & Controles & 79 & 49,10 & 45,31 & 53,47 & \\
\hline \multirow{2}{*}{ Tiempo en seguimiento (años) } & Casos & 40 & 17,50 & 9,75 & 22,00 & \multirow{2}{*}{0,171} \\
\hline & Controles & 78 & 15,00 & 8,00 & 21,00 & \\
\hline \multirow{2}{*}{ Tiempo en tratamiento (años) } & Casos & 40 & 17,00 & 8,25 & 20,00 & \multirow{2}{*}{0,094} \\
\hline & Controles & 78 & 13,00 & 5,00 & 19,00 & \\
\hline \multirow{2}{*}{ Número de pastillas/día } & Casos & 40 & 3,00 & 3,00 & 4.00 & \multirow{2}{*}{0,001} \\
\hline & Controles & 79 & 2,00 & 1,00 & 3.00 & \\
\hline
\end{tabular}

Tabla 5. Consumo de sustancias y significación estadística de la comparación. Casos y controles.

\begin{tabular}{lcccc}
\hline & Controles & \multicolumn{2}{c}{ Casos } & \multicolumn{2}{c}{ Prueba de Pearson } \\
\cline { 2 - 5 } Consumo Alcohol & $\mathrm{n}(\%)$ & $\mathrm{n}(\%)$ & $\chi^{2}$ & Sig. \\
\hline Consumo Cannabis & $12(15,8)$ & $15(37,5)$ & 6,917 & 0,009 \\
\hline Consumo Cocaína & $24(30,4)$ & $15(37,5)$ & 0,611 & 0,434 \\
\hline Consumo Heroína & $13(16,5)$ & $16(40,0)$ & 7,987 & 0,005 \\
\hline Consumo Metadona & $3(3,8)$ & $5(12,5)$ & 3,207 & 0,073 \\
\hline Policonsumo & $5(6,3)$ & $12(30,0)$ & 12,151 & 0,000 \\
\hline
\end{tabular}


Tabla 6. Regresión logística, método 'introducir'.

\begin{tabular}{lccccccc}
\hline & B & E.T. & Wald & gl & Sig. & Exp(B) & IC 95\% \\
\hline Edad & $-0,092$ & 0,044 & 4,348 & 1 & 0,037 & 0,912 & $0,837-0,995$ \\
\hline Género & $-1,346$ & 0,612 & 4,834 & 1 & 0,028 & 0,260 & $0,078-0,864$ \\
\hline Tiempo seguimiento & 0,063 & 0,041 & 2,293 & 1 & 0,130 & 1,065 & $0,982-1,154$ \\
\hline No pastillas/día & 0,515 & 0,203 & 6,469 & 1 & 0,011 & 1,674 & $1,126-2,491$ \\
\hline Nivel socioeconómico & 1,347 & 0,581 & 5,374 & 1 & 0,020 & 3,844 & $1,231-12,001$ \\
\hline Consumo Alcohol & 1,465 & 0,673 & 4,735 & 1 & 0,030 & 4,330 & $1,157-16,206$ \\
\hline Consumo Cannabis & $-0,646$ & 0,588 & 1,209 & 1 & 0,272 & 0,524 & $0,165-1,659$ \\
\hline Consumo Cocaína & 0,380 & 0,715 & 0,283 & 1 & 0,595 & 1,463 & $0,360-5,943$ \\
\hline Consumo Metadona & 1,624 & 0,801 & 4,112 & 1 & 0,043 & 5,074 & $1,056-24,379$ \\
\hline Consumo Heroína & $-0,517$ & 1,088 & 0,226 & 1 & 0,635 & 0,596 & $0,071-5,035$ \\
\hline Constante & $-2,282$ & 3,062 & 0,556 & 1 & 0,456 & 0,102 & \\
\hline
\end{tabular}

o consumo no perjudicial una vez ajustado por las variables señaladas. El uso de metadona incrementa este riesgo en 5,074 (IC95\%: 1,056-24,379). En cambio, los consumos de cannabis, cocaína o heroína no se asocian significativamente con un empeoramiento en la adherencia terapéutica.

\section{Datos sociodemográficos y clínicos.}

Según los resultados, aumentan el riesgo de no adherencia terapéutica el género masculino, una peor situación socioeconómica, así como un régimen de tratamiento antirretroviral consistente en un mayor número de pastillas al día.

\section{Discusión}

De lo expuesto se desprende que el consumo perjudicial de alcohol empeora significativamente la adherencia al tratamiento antirretroviral. Esto suscribe en nuestro entorno asistencial y cultural lo señalado amplia y consistentemente en la literatura previa en otros contextos (Azar et al., 2010; Vagenas et al., 2015; Hendershot et al., 2009). En el presente estudio, además, la asociación entre el consumo de alcohol y la falta de adherencia se mantiene tras el ajuste por otros consumos por lo que, en la muestra estudiada, el alcohol deteriora la adherencia de manera independiente al uso o no de otras sustancias.

Se evidencia también una influencia negativa del uso de metadona sobre la adherencia, existiendo dudas sobre el sentido de este resultado. El uso terapéutico de la misma, al menos en nuestro país, podría corresponderse con sujetos clínicamente más deteriorados o con un perfil socioeconómico más bajo (Ladero, Orejudo y Carrobles, 2005), lo que explicaría una peor adherencia y resultaría congruente con los resultados obtenidos. No obstante, estudios previos han planteado que la pertenencia a un programa terapéutico con metadona, frente a usuarios de drogas por vía parenteral sin seguimiento, podría suponer una mejora en la adherencia al tratamiento antirretroviral (Malta, Strathdee, Magnanini y Bastos, 2008), sobre todo en zonas marginales con elevada prevalencia de este tipo de consumo (Azar et al., 2015).

Por otro lado, en el presente estudio no se ha hallado asociación significativa entre el consumo de cannabis, cocaína o heroína y el deterioro de la adherencia terapéutica, lo cual difiere globalmente de los hallazgos publicados previamente en la literatura en otros contextos (Hinkin et al., 2004; Azar et al., 2015). No obstante, la ausencia de relación entre el consumo de cannabis y el incumplimiento terapéutico ya ha sido señalada previamente por otros autores (Rosen et al., 2013; De Jong, Prentiss, McFarland, Machekano e Israelski, 2005). En esta muestra, además, no existen diferencias significativas en el análisis bruto entre casos y controles en dicho consumo. Respecto al uso de heroína o cocaína, se estima que la ausencia de relación estadísticamente significativa podría deberse al escaso número de sujetos que reconocen dicho consumo, aunque existe controversia sobre la capacidad de reporte del consumo de los pacientes (Van Dorn, Desmarais, Swartz, Young y Sellers, 2014).

De los resultados expuestos se extrae que los sujetos con peor situación socioeconómica presentan más riesgo de incumplimiento terapéutico. Esto sería congruente con otros hallazgos descritos en la literatura. Se sabe que la existencia de soporte social estructurado y domicilio fijo facilitan la adherencia (Ruiz-Pérez et al., 2006), lo cual subraya la importancia de los factores psicosociales sobre el estado global de salud y la necesidad de ahondar en la investigación en este campo, que ha cobrado importancia en los últimos años (Ruiz-Pérez et al., 2006; Beer, Mattson, Bradley y Skarbinski, 2016).

En el grupo de sujetos clasificados como no adherentes, existe un mayor porcentaje de supervisión del tratamien- 
to por parte de terceros, que probablemente deba ser entendida como una consecuencia de la situación basal de incumplimiento. La superioridad -en términos de control inmunológico de la infección y adherencia- de la administración supervisada de la medicación en consumidores de sustancias respecto a la toma de medicación autónoma ha sido demostrada tanto en estudios aleatorizados como en revisiones sistemáticas (Binford, Kahana y Altice, 2012).

Diferentes autores ya habían señalado que los regímenes de tratamiento más complicados (mayor número de pastillas al día) empeoran la adherencia al tratamiento (Ammassari et al., 2002; Stone, Jordan, Tolson, Miller y Pilon, 2004; Nachega et al., 2014).

El presente estudio corrobora en nuestro entorno gran parte de los hallazgos publicados en la literatura de forma previa. Cabe destacar, a su favor, la fiabilidad de la adherencia terapéutica -en la que el reporte objetivo y cuantitativo de Farmacia Hospitalaria viene consolidado por el instrumento SMAQ y la opinión del profesional de referencia-, así como la inclusión de distintas variables de consumo y sociodemográficas.

Entre las limitaciones del trabajo ha de señalarse el acotamiento en la potencia del estudio que ejerce el tamaño muestral, así como la baja incidencia en el consumo de heroína y cocaína, lo cual parece limitar en esta muestra el papel que dichas variables pueden ejercer en realidad. Cabe destacar que los datos sobre la historia de consumo de sustancias se obtuvieron únicamente mediante entrevista con el paciente. Además, atendiendo a los requerimientos de un seguimiento ambulatorio regular, es probable que sujetos con patrones de consumo más graves y marcadores más severos de deterioro social y clínico hayan quedado al margen de este estudio. Se debe asumir que los resultados obtenidos son aplicables y extrapolables solo a aquellas poblaciones que compartan un perfil similar. Sería recomendable, en este sentido, ampliar el estudio a poblaciones extraídas de ambientes con mayor dificultad de acceso a los cuidados de salud, patrones de consumo más severos o mayor deterioro psicosocial, para estudiar la repercusión del consumo de alcohol y otras sustancias en la adherencia terapéutica en sujetos con este perfil.

El consumo de sustancias fue medido de manera cualitativa -siguiendo los criterios de la OMS para consumo perjudicial en el caso del alcohol-, y cuantitativa, sin estudiar si los pacientes cumplían criterios para las categorías diagnósticas de abuso o dependencia de sustancias. No obstante, considerar los daños relacionados con el uso de sustancias dentro de un continuum y no como entidades estáticas, aumentaría la sensibilidad en la detección de consumos de riesgo para la no adherencia, y es la línea que sigue el actual DSM-5.

Procede resaltar que la estimación de la adherencia según reporte de Farmacia Hospitalaria se basa en la retirada o no de la medicación, y no estrictamente en su consumo.
A esto se añaden otras limitaciones como la incapacidad para identificar defectos en el horario de las tomas, la compensación con tomas extra o el extravío de pastillas (Berg y Arnsten, 2006). No obstante, este método se ha demostrado altamente específico aunque poco sensible, por lo que la utilización de métodos complementarios en el presente estudio disminuye el riesgo de una posible sobreestimación de la adherencia (Henegar et al., 2015).

Entre las limitaciones del estudio, por último, cabe senalar que en el análisis de la muestra que presentamos no se han incluido variables relacionadas con la presencia o no de psicopatología (Torrens, Mestre-Pintó, Montanari y Vicente, 2017), o con el estado cognitivo de los sujetos que la conforman. Debe tenerse en cuenta, también, la posibilidad de sesgos de selección y supervivencia inherentes al diseño de estudio utilizado. Ningún paciente fue excluido por no consentimiento. No se recogieron los datos referentes a pacientes excluidos por otras causas.

Es sabido que la adherencia al tratamiento antirretroviral posee un papel fundamental en la morbimortalidad prevenible en pacientes VIH+ (Braithwaite y Bryant, 2010). A la luz de los resultados obtenidos es esencial, por tanto, abordar la posible existencia de consumo de sustancias -y de manera especial de alcohol- en el marco clínico cotidiano, interrogando sobre el tipo, frecuencia y cantidad de sustancia consumida a fin de poder llevar a cabo medidas que disminuyan el impacto del consumo en el cumplimiento terapéutico (Parsons et al., 2014). Además de incidir en la detección de posibles patrones de riesgo, sería pertinente facilitar a los pacientes consumidores el acceso a recursos específicos para el tratamiento de dicha problemática (Gonzalez et al., 2013). En base a lo expuesto, los factores comportamentales, estructurales, sociales o psicológicos relacionados con la adherencia en los diferentes grupos de consumidores de sustancias deberían centrar nuevas investigaciones a fin de desarrollar intervenciones específicas (Azar et al., 2015).

\section{Conclusiones}

El estudio pone de manifiesto la importancia del consumo de alcohol como factor que incide negativamente sobre la adherencia al tratamiento antirretroviral. Esta importancia se mantiene aun considerando el consumo de otras sustancias, y el ajuste por otras variables que influyen en la adherencia, tales como el género masculino, una situación socioeconómica problemática o un número elevado de pastillas en la pauta prescrita. La asunción de la adherencia terapéutica como un proceso dinámico y modificable justifica la intervención a distintos niveles a fin de minimizar los factores asociados al empeoramiento de la misma. Explorar detenidamente el consumo de alcohol (y de otras sustancias) se hace imprescindible en el abordaje de pacientes con VIH y tratamiento antirretroviral de 
gran actividad (TARGA). De esta forma, podría facilitarse la promoción de una mejor evolución de la enfermedad, con disminución de la morbimortalidad y del riesgo de transmisión del virus, así como del desarrollo de cepas resistentes. En estudios con sujetos $\mathrm{VIH}+$ es necesario considerar la gran heterogeneidad existente entre pacientes. Por ello, en la práctica clínica será sustancial individualizar cada caso e identificar qué factores, tanto de riesgo como de protección, pueden condicionar la adherencia al tratamiento antirretroviral.

\section{Conflicto de intereses}

Los autores declaran que no existe ningún conflicto de interés para el presente trabajo. Enriqueta Ochoa Mangado declara que en los últimos años ha recibido financiación como ponente y ha colaborado en proyectos de Lundbeck, Servier, Reckitt Benckiser/Indivior, y Ferrer-Brainfarma.

\section{Referencias}

Ammassari, A., Trotta, M.P., Murri, R., Castelli, F., Narciso, P, Noto, P., ... Antinori A. (2002). Correlates and predictors of adherence to highly active antiretroviral therapy: overview of published literature. Journal of Acquired Immune Deficiency Syndromes, 31, S123-127.

Anand, P., Springer, S.A., Copenhaver, M.M. y Altice, F.L. (2010). Neurocognitive impairment and HIV risk factors: a reciprocal relationship. AIDS and Behavior, 14, 1213-1226. doi:10.1007/s10461-010-9684-1.

Azar, M.M., Springer, S.A., Meyer, J.P. y Altice, F.L. (2010). A systematic review of the impact of alcohol use disorders on HIV treatment outcomes, adherence to antiretroviral therapy and health care utilization. Drug and Alcohol Dependence, 112, 178-193. doi:10.1016/j.drugalcdep.2010.06.014.

Azar, P., Wood, E., Nguyen, P., Luma, M., Montaner, J., Kerr, T. y Milloy, M.J. (2015). Drug use patterns associated with risk of non-adherence to antiretroviral therapy among HIV-positive illicit drug users in a Canadian setting: a longitudinal analysis. BMC Infectious Diseases, 15, 193. doi:10.1186/s12879-015-0913-0.

Baum, M.K., Rafie, C., Lai, S., Sales, S., Page, J.B. y Campa, A. (2010). Alcohol use accelerates HIV disease progression. AIDS Research and Human Retroviruses, 26, 511-518. doi:10.1089/aid.2009.0211.

Beer, L., Mattson, C.L., Bradley, H. y Skarbinski, J. (2016). Medical Monitoring Project. Understanding cross-sectional racial, ethnic, and gender disparities in antiretroviral use and viral suppression among HIV patients in the United States. Medicine, 95, e3171. doi:10.1097/ MD.0000000000003171.

Berg, K.M. y Arnsten, J.H. (2006). Practical and conceptual challenges in measuring antiretroviral adherence. Jour- nal of Acquired Immune Deficiency Syndromes, 43, S79-S87. doi:10.1097/01.qai.0000248337.97814.66.

Bermudez, L.G., Jennings, L., Ssewamala, F.M., Nabunya, P., Mellins, C. y McKay, M. (2016). Equity in adherence to antiretroviral therapy among economically vulnerable adolescents living with HIV in Uganda. AIDS Care, 28, 83-91. doi:10.1080/09540121.2016.1176681.

Binford, M.C., Kahana, S.Y. y Altice, F.L. (2012). A systematic review of antiretroviral adherence interventions for HIV-infected people who use drugs. Current HIV/AIDS Reports, 9, 287-312. doi:10.1007/s11904-012-0134-8.

Braithwaite, R.S. y Bryant, K.J. (2010). Influence of alcohol consumption on adherence to and toxicity of antiretroviral therapy and survival. Alcohol Research and Health, 33, 280-287.

Cohen, C.J. (2006). Successful HIV treatment: lessons learned. Journal of Managed Care $\mathcal{E}$ Specialty Pharmacy, 12, S6-S11. doi:10.18553/jmcp.2006.12.S7-B.S6

Cortés Tomás, M.T. y Motos Sellés, P. (2016). Cómo definir y medir el consumo intensivo de alcohol. En M.T. Cortés Tomás (Coord.) Consumo intensivo de alcohol en jóvenes. Guía Clínica (pp. 25-46), España: Socidrogalcohol.

De Jong, B.C., Prentiss, D., McFarland, W., Machekano, R. e Israelski, D.M. (2005). Marijuana use and its association with adherence to antiretroviral therapy among HIV-infected persons with moderate to severe nausea. Journal of Acquired Immune Deficiency Syndromes, 38, 43-46.

Galvan, F.H., Bing, E.G., Fleishman, J.A., London, A.S., Caetano, R., Burnam, M.A., ... Shapiro, M. (2002). The prevalence of alcohol consumption and heavy drinking among people with HIV in the United States: results from the HIV Cost and Services Utilization Study. Journal of Studies on Alcohol and Drugs, 63, 179-186.

Gonzalez, A., Barinas, J. y O'Cleirigh, C. (2011). Substance use: impact on adherence and HIV medical treatment. Current HIV/AIDS Reports, 8, 223-234. doi:10.1007/ s11904-011-0093-5.

Gonzalez, A., Mimiaga, M.J., Israel, J., Andres Bedoya, C. y Safren, S.A. (2013). Substance use predictors of poor medication adherence: the role of substance use coping among HIV-infected patients in opioid dependence treatment. AIDS and Behavior, 17, 168-173. doi:10.1007/ s10461-012-0319-6.

Hendershot, C.S., Stoner, S.A., Pantalone, D.W. y Simoni, J.M. (2009). Alcohol use and antiretroviral adherence: review and meta-Analysis. Journal of Acquired Immune Deficiency Syndromes, 52, 180-202. doi:10.1097/QAI.0b013e3181b18b6e.

Henegar, C.E., Westreich, D., Maskew, M., Brookhart, M.A., Miller, W.C., Majuba, P. y Van Rie, A. (2015). Comparison of pharmacy-based measures of adherence to antiretroviral therapy as predictors of virological failure. AIDS and Behaviour, 19, 612-618. doi:10.1007/s10461014-0953-2. 
Hinkin, C.H., Hardy, D.J., Mason, K.I., Castellon, S.A., Durvasula, R.S., Lam, M.N. y Stefaniak, M. (2004). Medication adherence in HIV-infected adults: effect of patient age, cognitive status, and substance abuse. AIDS, 18, S19-S25.

Hudelson, C. y Cluver, L. (2015). Factors associated with adherence to antiretroviral therapy among adolescents living with HIV/AIDS in low- and middle-income countries: a systematic review. AIDS Care, 27, 805-816. doi:10. 1080/09540121.2015.1011073.

Hutton, H.E. y Treisman, G. (2008). The Role of Personality in HIV Risk Behaviors: Implications for Treatment. En M.A. Cohen y J.M. Gorman (Ed.), Comprehensive Textbook of AIDS Psychiatry (pp. 141-149). Nueva York: Oxford University Press.

Instituto de Salud Carlos III, Área de Vigilancia de VIH y Comportamientos de Riesgo (2016). Vigilancia Epidemiológica del VIH y sida en España: Sistema de Información sobre Nuevos Diagnósticos de VIH y Registro Nacional de Casos de Sida. Plan Nacional sobre el Sida - S.G. de Promoción de la Salud y Epidemiología / Centro Nacional de Epidemiología - ISCIII. Madrid. Recuperado de https://www. msssi.gob.es/ciudadanos/enfLesiones/enfTransmisibles/sida/vigilancia/InformeVIH_SIDA_2016.pdf.

Kalichman, S.C., Kalichman, M.O., Cherry, C., Hoyt, G., Washington, C., Grebler, T., ... Merely, C. (2015). Intentional Medication Nonadherence Because of Interactive Toxicity Beliefs Among HIV-Positive Active Drug Users. Journal of Acquired Immune Deficiency Syndromes, 70, 503509. doi:10.1097/QAI.0000000000000776.

Knobel, H., Alonso, J., Casado, J.L., Collazos, J., González, J., Ruiz, I., ... Ocampo, A. (2002). Validation of a simplified medication adherence questionnaire in a large cohort of HIV-infected patients: the GEEMA Study. AIDS, 16, 605-613.

Ladero, L., Orejudo, S. y Carrobles, J.A. (2005). Variables psicosociales en la adherencia al tratamiento antirretroviral en pacientes adscritos a un programa de mantenimiento con metadona. Psicothema, 17, 575-581.

Lazo, M., Gange, S.J., Wilson, T.E., Anastos, K., Ostrow, D.G., Witt, M.D. y Jacobson, L.P. (2007). Patterns and predictors of changes in adherence to highly active antiretroviral therapy: longitudinal study of men and women. Clinical Infectious Diseases, 45, 1377-1385. doi:10.1086/522762.

Lucas, G.M., Gebo, K.A., Chaisson, R.E. y Moore, R.D. (2002). Longitudinal assessment of the effects of drug and alcohol abuse on HIV-1 treatment outcomes in an urban clinic. AIDS, 16, 767-774.

Malta, M., Strathdee, S.A., Magnanini, M.M.F. y Bastos, F.I. (2008). Adherence to antiretroviral therapy for human immunodeficiency virus/acquired immune deficiency syndrome among drug users: a systematic review. Addiction, 103, 1242-1257. doi:10.1111/j.13600443.2008.02269.x.
Nachega, J.B., Parienti, J.J., Uthman, O.A., Gross, R., Dowdy, D.W., Sax, P.E., ... Giordano, T.P. (2014). Lower pill burden and once-daily antiretroviral treatment regimens for HIV infection: A meta-analysis of randomized controlled trials. Clinical Infectious Diseases, 58, $1297-$ 1307. doi:10.1093/cid/ciu046.

Observatorio Español de la Droga y las Toxicomanías (OEDT), Delegación del Gobierno para el Plan Nacional sobre Drogas (DGPNSD) y Ministerio de Sanidad, Servicios Sociales e Igualdad (MSSS), (2015). EDADES. Encuesta sobre Alcohol y Drogas en España. Recuperado de http:/ / www.pnsd.msssi.gob.es/ca/profesionales/sistemasInformacion/sistemaInformacion/pdf/2015_Informe_EDADES.pdf.

Organización Mundial de la Salud (O.M.S), (1992). CIE10. Décima Revisión de la Clasificación Internacional de Enfermedades. Trastornos Mentales y del Comportamiento. Madrid: Meditor.

Ortego, C., Huedo-Medina, T.B., Vejo, J. y Llorca, F.J. (2011). Adherence to highly active antiretroviral therapy in Spain: A meta-analysis. Gaceta Sanitaria, 25, 282328. doi:10.1016/j.gaceta.2010.10.016.

Palepu, A., Horton, N.J., Tibbetts, N., Meli, S. y Samet, J.H. (2004). Uptake and adherence to highly active antiretroviral therapy among HIV-infected people with alcohol and other substance use problems: the impact of substance abuse treatment. Addiction, 99, 361-368.

Parry, C. D., Morojele, N. K., Myers, B. J., Kekwaletswe, C. T., Manda, S. O., Sorsdahl, K., ... Shuper, P. A. (2014). Efficacy of an alcohol-focused intervention for improving adherence to antiretroviral therapy (ART) and HIV treatment outcomes - a randomised controlled trial protocol. BMC Infectious Diseases, 14, 500. doi:10.1186/14712334-14-500.

Parsons, J.T., Starks, T.J., Millar, B.M., Boonrai, K. y Marcotte, D. (2014). Patterns of substance use among HIV-positive adults over 50: implications for treatment and medication adherence. Drug and Alcohol Dependence, 139, 33-40. doi:10.1016/j.drugalcdep.2014.02.704.

Paterson, D.L., Swindells, S., Mohr, J., Brester, M., Vergis, E.N., Squier, C.,... Singh,N. (2000). Adherence to protease inhibitor therapy and outcomes in patients with HIV infection. Annals of Internal Medicine, 133, 21-30.

Peltzer, K. y Pengpid, S. (2013). Socioeconomic factors in adherence to HIV therapy in low- and middle-income countries. Journal of Health, Population and Nutrition, 31, 150-170.

Pérez-Valero, I., Hontañón-Antoñana, V., Cáceres, G., Montes, J., Montes-Ramírez, M., Bernardino, I., ... González Baeza, A. (2016a). High rates of alcohol consumption among HIV-infected patients and poorer ART adherence in those patients with risky alcohol intake. Póster presentado al VIII Congreso Nacional Grupo de Estudio de Sida. San Sebastián, España. Recuperado de https://intranet.pa- 
cifico-meetings.com/amsysweb/faces/publicacionOnline.xhtml?id=324.

Pérez-Valero, I., Hontañón-Antoñana, V., Montes, J., Cáceres, G., Valencia, E., Martín-Carbonero, L.,... González Baeza, A. (2016b). Poorer antiretroviral treatment outcomes and higher anxiety levels associated to illicit drug consumption among HIV-infected patients attended in Spain. Póster presentado al VIII Congreso Nacional Grupo de Estudio de Sida. San Sebastián, España. Recuperado de https:// intranet.pacifico-meetings.com/amsysweb/faces/publicacionOnline. $x$ html id $=324$.

Poorolajal, J., Hooshmand, E., Mahjub, H., Esmailnasab, N. y Jenabi, E. (2016). Survival rate of AIDS disease and mortality in HIV-infected patients: a meta-analysis. Public Health, 139, 3-12. doi:10.1016/j.puhe.2016.05.004.

Rosen, M.I., Black, A.C., Arnsten, J.H., Goggin, K., Remien, R.H., Simoni, J.M., ... Liu, H. (2013). Association between use of specific drugs and antiretroviral adherence: findings from MACH 14. AIDS and Behavior, 17, 142147. doi:10.1007/s10461-011-0124-7.

Ruiz-Pérez, I., Olry de Labry-Lima, A., Prada-Pardal, J.L., Rodríguez-Baño, J., Causse-Prados, M., López-Ruz, M.A., ... Morales-Rojas, D. (2006). Impacto de los factores demográficos y psicosociales en la no adherencia a los fármacos antirretrovirales. Enfermedades Infecciosas y Microbiología Clínica, 24, 373-378.

Scott-Sheldon, L. A. J., Walstrom, P., Carey, K. B., Johnson, B. T., Carey, M. P. y the MASH Research Team. (2013). Alcohol Use and Sexual Risk Behaviors among Individuals Infected with HIV: A Systematic Review and Meta-Analysis 2012 to Early 2013. Current HIV/AIDS Reports, 10, 314-323. doi:10.1007/s11904-013-0177-5.

Skalski, L.M., Sikkema, K.J., Heckman, T.G. y Meade, C.S. (2013). Coping styles and illicit drug use in older adults with HIV/AIDS. Psychology of Addictive Behaviors, 27, 1050-1058. doi:10.1037/a0031044.

Stone, V.E., Jordan, J., Tolson, J., Miller, R. y Pilon, T. (2004). Perspectives on adherence and simplicity for HIV-infected patients on antiretroviral therapy: self-report of the relative importance of multiple attributes of highly active antiretroviral therapy (HAART) regimens in predicting adherence. Journal of Acquired Immune Deficiency Syndromes, 36, 808-816.

Tancredi, M.V. y Waldman, E.A. (2014). Survival of AIDS patients in Sao Paulo-Brazil in the pre- and post-HAART eras: a cohort study. BMC Infectious Diseases, 14, 599. doi:10.1186/s12879-014-0599-8.

Taylor, A.L., Denniston, M.M., Klevens, R.M., McKnight-Eily, L.R. y Jiles, R.B. (2016). Association of Hepatitis C Virus with Alcohol Use Among U.S. Adults: NHANES 2003-2010. American Journal of Preventive Medicine, 51, 206-215. doi:10.1016/j.amepre.2016.02.033.

Torrens, M., Mestre-Pintó, J.I., Montanari, L., Vicente, J. y Domingo-Salvany, A. (2017). Dual diagnosis: an Euro- pean perspective. Adicciones, 29, 3-5. doi:10.20882/adicciones.933.

Tripathi, A., Youmans, E., Gibson, J.J. y Duffus, W.A. (2011). The impact of retention in early HIV medical care on viro-immunological parameters and survival: a statewide study. AIDS Research and Human Retroviruses, 27, 751-758. doi:10.1089/AID.2010.0268.

Tucker, J.S, Orlando, M., Burnam, M.A., Sherbourne, C.D., Kung, F.Y. y Gifford, A.L. (2004). Psychosocial mediators of antiretroviral nonadherence in HIV-positive adults with substance use and mental health problems. Health Psychology, 23, 363-370. doi:10.1037/0278-6133.23.4.363.

Vagenas, P., Azar, M.M., Copenhaver, M.M., Springer, S.A., Molina, P.E. y Altice, F.L. (2015). The impact of alcohol use and related disorders on the HIV continuum of care: a systematic review: alcohol and the HIV continuum of care. Current HIV/AIDS Reports, 12, 421-436. doi:10.1007/s11904-015-0285-5.

Van Dorn, R. A., Desmarais, S. L., Swartz, M. S., Young, M. S. y Sellers, B. G. (2014). Letter to the Editor: Critique of Bahorik et al. (2013) - "Under-reporting of drug use among individuals with schizophrenia: prevalence and predictors." Psychological Medicine, 44, 668-670. doi:10.1017/S0033291713002511.

Viswanathan, S., Justice, A.C., Alexander, G.C., Brown, T.T., Gandhi, N.R., McNicholl, I.R., ... Jacobson, L.P. (2015). Adherence and HIV RNA Suppression in the Current Era of Highly Active Antiretroviral Therapy. Journal of Acquired Immune Deficiency Syndromes, 69, 493-498. doi:10.1097/QAI.0000000000000643.

Williams, E.C., Hahn, J.A., Saitz, R., Bryant, K., Lira, M.C. y Samet, J.H. (2016). Alcohol Use and Human Immunodeficiency Virus (HIV) Infection: Current Knowledge, Implications, and Future Directions. Alcoholism: Clinical and Experimental Research, 40, 2056-2072. doi:10.1111/ acer.13204.

Zarkin, G.A., Bray, J.W., Babor, T.F. y Higgins-Biddle, J.C. (2004). Alcohol drinking patterns and health care utilization in a managed care organization. Health Services Research, 39, 553-570. doi:10.1111/j.14756773.2004.00244.x. 